Supporting Information

\title{
Oxophilicity as a Descriptor for NO Cleavage Efficiency over Group IX Metal Clusters
}

\author{
Masato Yamaguchi ${ }^{\dagger}$, Yufei Zhang ${ }^{\dagger}$, Satoshi Kudoh ${ }^{\dagger}$, Kohei Koyama ${ }^{\dagger}$, Olga V. \\ Lushchikova ${ }^{\ddagger}$, Joost M. Bakker ${ }^{\ddagger}$ Fumitaka Mafuné ${ }^{* \dagger}$ \\ $\dagger$ Department of Basic Science, School of Arts and Sciences, The University of Tokyo, \\ Komaba, Meguro, Tokyo 153-8902, Japan \\ $\$$ Radboud University, Institute for Molecules and Materials, FELIX Laboratory, 6525 \\ ED Nijmegen, Netherlands
}

\section{Experimental Methods}

Geometries of cationic $\mathrm{Ir}_{6}{ }^{+} \mathrm{NO}$ clusters were investigated by IR multiple-photon dissociation (IRMPD) spectroscopy of their complexes with $\mathrm{Ar}\left(\operatorname{Ir}_{6}{ }^{+} \mathrm{NO}-\mathrm{Ar}\right)$ employing the IR free-electron laser FELIX. Clusters were produced using laser ablation of a solid Ir rod (purity: $99.95 \%$ ) by the second harmonic of a Nd:YAG laser (532 nm) which operated at $20 \mathrm{~Hz}$ and a typical pulse energy of $\sim 10 \mathrm{~mJ}$, in the presence of an $\mathrm{Ar} / \mathrm{He}$ mixture (approx. $0.43 \% \mathrm{Ar}, 0.8 \mathrm{MPa}$ stagnation pressure) injected into clustering channel using a pulsed valve. Generated clusters were reacted with a $\mathrm{NO} / \mathrm{He}$ reactant gas mixture (approx. $11.11 \% \mathrm{NO})$ which was introduced via a second plused valve $(0.1 \mathrm{MPa}$ stagnation pressure) about $30 \mathrm{~mm}$ downstream from the laser ablation point. To enhance the attachment of Ar to the cluster, reaction cell was cooled down to temperature of $\sim 200$ $\mathrm{K}$.

After formation, the reactive mixture was expanded into vacuum forming a molecular beam which was collimated by a $2 \mathrm{~mm}$ diameter skimmer, and a $1 \mathrm{~mm}$ diameter aperture before entering the source region of a reflectron time-of-flight mass spectrometer. 
Prior to extraction by two high-voltage plates, the ions are irradiated by one macropulse of the FELIX laser beam, that was attenuated by fixed value mesh attenuators to prevent saturation of the strongest bands. The experiment was operated at twice the FELIX repetition rate allowing for the recording of reference mass spectra which were used to correct for source fluctuations.

\section{Computational methods}

The stable structures of $\operatorname{Ir}_{6} \mathrm{NO}^{+}$were calclated by quantum chemical calculations using Gaussian 09. Computational procedures were almost same as the one described in our previous article. ${ }^{1}$ The density functional theory (DFT) was used for whole calclations of not only motief and viblational freqencies but also the reaction path. To make a complete search of the stable isomers with reduced computational costs, initial geometries, prepared manually and randomly, were optimized, at first, with the LanL2DZ and the 6$31 \mathrm{G}(\mathrm{d})$, for Ir and N, O atoms respectively for the conceivable spin states (see Figure $\mathrm{S} 1$ ). For the obtained low energy isomers, the geometris were re-optimized using the Becke's three-parameter hybrid density functional with the Lee-Yang-Parr correlation functional (B3LYP) with the Stuttgart/Dresden (SDD, MWB60) basis set and effective core potensial (ECP) for Ir atoms and the $6-311+\mathrm{G}(\mathrm{d})$ basis set for $\mathrm{N}$ and $\mathrm{O}$ atoms.

Figure 4 in the article, is comparison of the binding energy of the $\mathrm{O}$ atom adsorbed on an on-top site of $\mathrm{Rh}_{6}{ }^{+}, \mathrm{Ir}_{6}{ }^{+}$and $\mathrm{Rh}_{5} \mathrm{Ta}^{+}$clusters. B3LYP with SDD and 6$311+\mathrm{G}(\mathrm{d})$ were used for optimization, and the zero point energies were included. Those configurations are in their global minimum with the conceivable spin states except for $\mathrm{Rh}_{6}{ }^{+} \mathrm{O}$. Most stable $\mathrm{Rh}_{6}{ }^{+} \mathrm{O}$ cluster possesses $\mathrm{O}$ atom at a hollow site surrounded by three $\mathrm{Rh}$ atoms with the 12tet spin state. 


\section{Isomeric ratios}

Isomeric ratios in the main text were estimated by comparison between the experimental band intensities and the computational IR intensities. Each band appearing in the IRMPD spectrum was assigned to a vibration of the isomers A, B and C. A band intensity was obtained from the spectral area of the band. The isomeric ratios were calculated based on the values of the band intensity divided by the IR intensity of the corresponding vibration calculated by Gaussian 09. Although observed IRMPD intensities do not necessarily scale linearly with IR laser pulse energy, such a linear power dependence does exist provided the threshold is low. ${ }^{2}$ For our studies, we employ Ar tagging, with binding energies of ca $0.1 \mathrm{eV},{ }^{3}$ which ensures we are as close to the linear regime as possible.

To further reduce the effects of a potential non-linear relationship between Ar elimination and IR absorption cross-section, we compared several combinations of assigned bands to calculate the isomeric ratio. For example, to obtain the ratios of molecularly to dissociatively attached clusters, we not only compared the main bands (950 vs $1815 \mathrm{~cm}^{-1}$ ) but also weaker bands $\left(550,600,1050 \mathrm{vs} 350 \mathrm{~cm}^{-1}\right.$ ), and their combinations $\left(550,600,1050\right.$ vs $\left.1815 \mathrm{~cm}^{-1}\right)$ and $\left(950\right.$ vs $\left.350 \mathrm{~cm}^{-1}\right)$. The standard deviation between the ratios obtained in the various ways is lower than $20 \%$, thus ruling out large non-linear effects.

(1) Yamaguchi, M.; Kudoh, S.; Miyajima, K.; Lushchikova, O. V.; Bakker, J. M.; Mafuné,

F. Tuning the Dissociative Action of Cationic Rh Clusters Toward NO by Substituting a Single Ta Atom. J. Phys. Chem. C 2019, 123 (6), 3476-3481. 
(2) Berden, G.; Derksen, M.; Houthuijs, K. J.; Martens, J.; Oomens, J. An Automatic Variable Laser Attenuator for IRMPD Spectroscopy and Analysis of Power-dependence in Fragmentation Spectra. Int. J. Mass Spectrom. 2019, 443, 1-8.

(3) Nagata, T.; Koyama, K.; Kudoh, S.; Miyajima, K.; Bakker, J. M.; Mafuné, F. Adsorption Forms of NO on $\mathrm{Rh}_{n}{ }^{+}(n=6-16)$ Revealed by Infrared Multiple Photon Dissociation Spectroscopy. J. Phys. Chem. C 2017, 121 (49), 27417-27426.

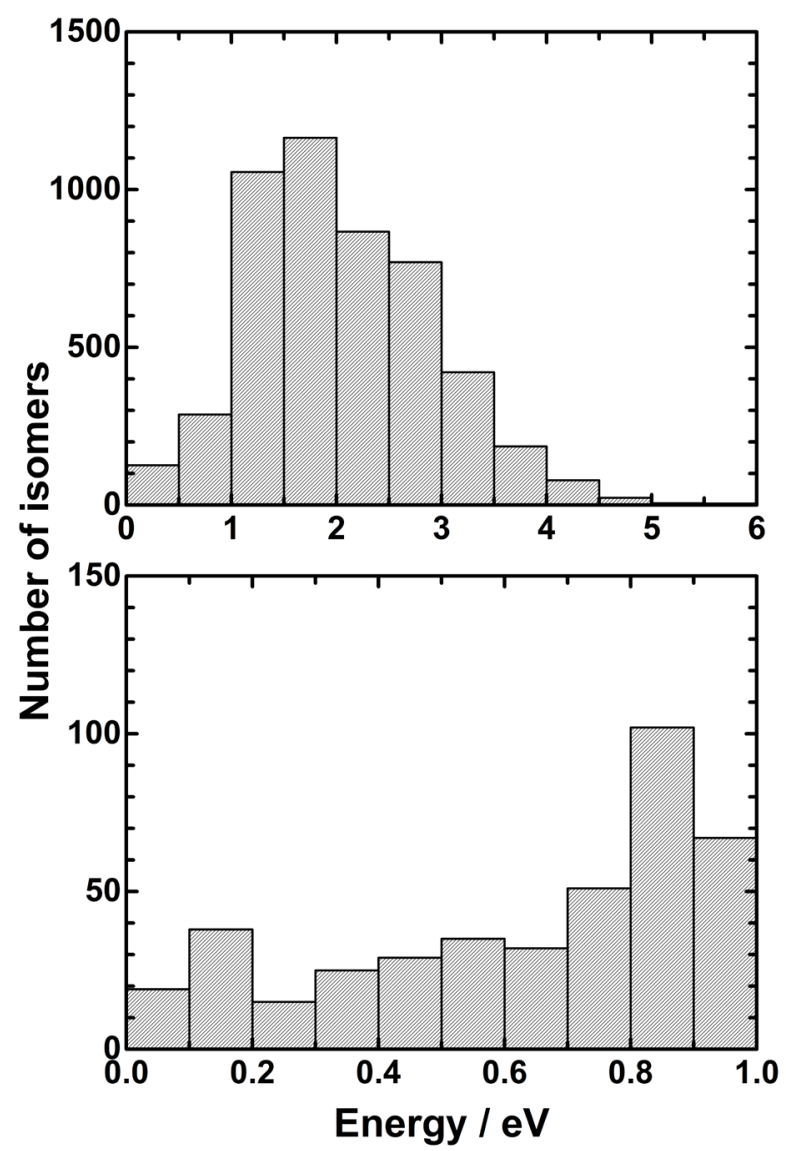

Figure S1 (a) Histogram showing the number of isomers obtained by the initial optimization for $\operatorname{Ir}_{6}{ }^{+} \mathrm{NO}$ by the DFT calculations using LanL2DZ basis sets from randomly set 5000 initial geometries, which is plotted against the difference in the formation energy from the most stable isomer. (b) Expanded histogram of (a) in the $0-1$ $\mathrm{eV}$ energy range for each $0.1 \mathrm{eV}$. 

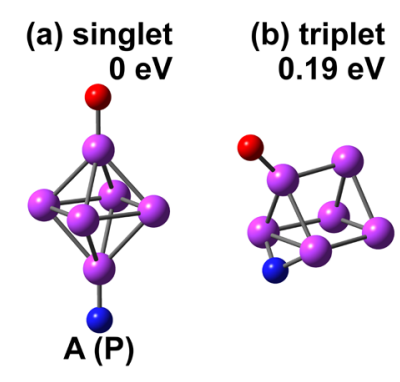
(c) singlet
$0.25 \mathrm{eV}$
(d) triplet
$0.29 \mathrm{eV}$

(e) quintet

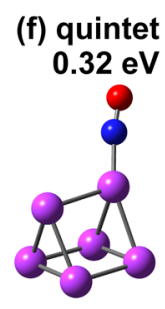

B (IM1)

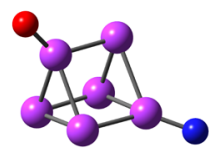

(IM5)

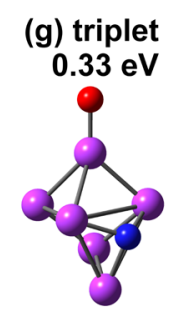

(h) triplet

$0.35 \mathrm{eV}$

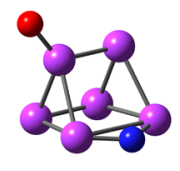

C (IM4)

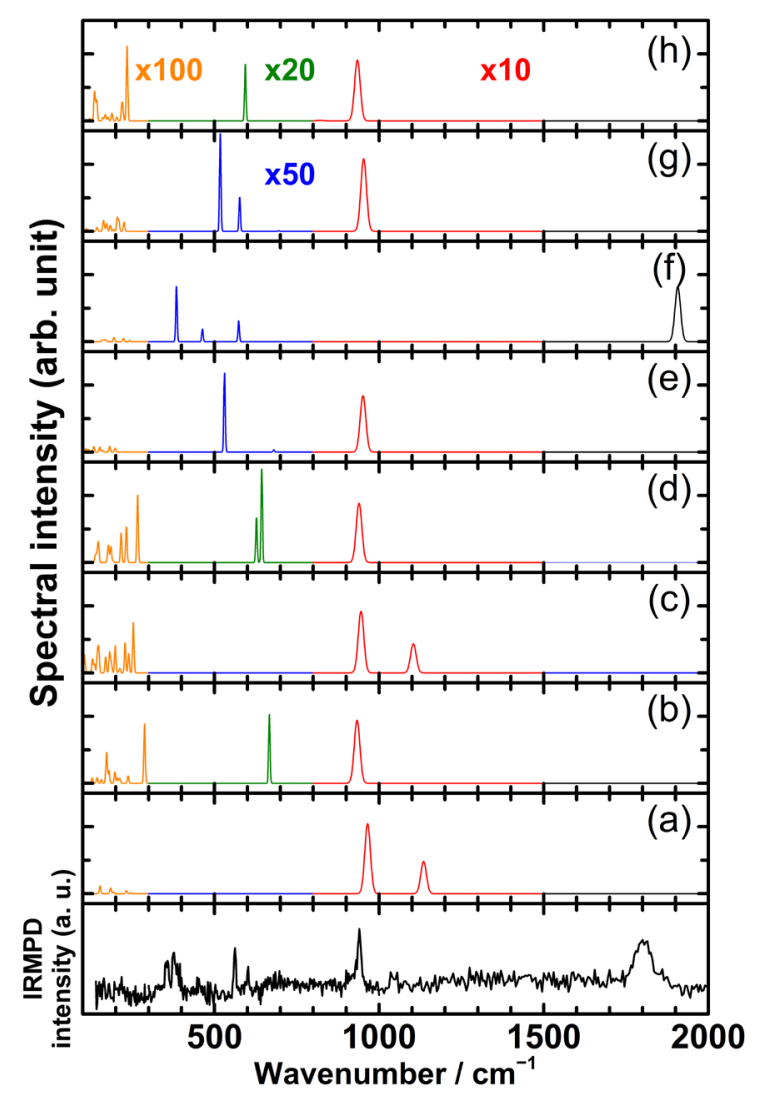

Figure S2 $(\mathrm{a}-\mathrm{h})$ Stable isomers of $\operatorname{Ir}_{6}{ }^{+} \mathrm{NO}$ and their vibrational spectra obtained by DFT calculations and IRMPD spectrum of $\operatorname{Ir}_{6}{ }^{+} \mathrm{NO}-\mathrm{Ar}$. Geometrical structures that are very similar to those appearing in Figure 2, but differing in spin, are nevertheless displayed using the same labels as in Figure 2, even though calculations displayed in Figure 2 were performed in the quintet spin state only. 